\title{
Repetitive Ureteral Stenting for Management of Transplant Graft Ureteral Obstruction
}

\author{
Lester S. Borden Jr, Vernon M. Pais Jr, Dean G. Assimos \\ Department of Urology, Wake Forest University School of Medicine, Winston-Salem, North \\ Carolina, USA
}

\begin{abstract}
Purpose: To review the use of repetitive stenting in the management of patients with ureteral obstruction after renal transplantation, with an emphasis on technique and functional graft outcome.

Materials and Methods: Five adult renal allograft recipients with ureteral obstruction were managed with repetitive ureteral stenting. Their hospital records, office notes, and operative reports were reviewed.

Results: All patients were successfully managed with retrograde ureteral stenting. They underwent an average of 8.8 stent changes over a mean 34.5 month follow up. No decline in renal function was observed.

Conclusions: Repetitive stenting is a viable treatment option for select patients with renal allograft ureteral obstruction.
\end{abstract}

Key words: kidney transplantation; ureteral obstruction; catheterization; stent; graft survival

Int Braz J Urol. 2006; 32: 142-6

\section{INTRODUCTION}

The most common urological complication following renal transplantation is ureteral obstruction with a reported incidence of 3-10\% (1-3). Percutaneous drainage with or without antegrade ureteral stent placement provides a temporary solution for such problems. Open surgical reconstruction is often the preferred method for correcting this problem. However, there are patients who either fail an open surgical reconstructive or endourologic approaches, or who are deemed not to be good candidates for these procedures. Repetitive ureteral stenting may be a viable option in this setting. Herein, we present our experience with this form of management.

\section{MATERIALS AND METHODS}

From November 1997 to April 2003, 460 renal transplant procedures and 26 combined pancreas and renal transplant operations were performed at the Wake Forest University Baptist Medical Center. Five adult male patients were diagnosed with ureteral obstruction following renal transplantation and were managed with repetitive ureteral stenting. The mean patient age was 50.4 years (range 27-67). Ureteral obstruction was diagnosed by new onset hydronephrosis demonstrated by ultrasonography and increasing serum creatinine levels. Three patients received deceased donor renal transplants, one received a living-related transplant, and one received a living-unrelated transplant. Three had failed attempts at open surgical reconstruction, Boari flap reconstruction in 1 and uretero-pyelostomy with native ureter in 2 , and declined further attempts at open surgical repair. Two had strictures longer than $2 \mathrm{~cm}$, were not thought to be good candidates for an endourological approach and declined open surgical repair. Three patients underwent initial endoscopic retrograde stent placement and two had initial percutaneous nephrostomy drain- 
age followed by antegrade stent placement. All subjects were then managed with retrograde stent changes. It is necessary to explain why some patients were poor candidates for such repair. Why did not you try nay endoscopic method like dilation or cold knife incision or laser incision?

The following approach was employed. Patients were administered prophylactic antibiotics prior to the induction of general or regional anesthesia. They were placed in the dorsal lithotomy position and cystoscopic removal of the indwelling ureteral stent was performed. The transplant ureteral orifice was identified and cannulated with a $5 \mathrm{~F}$ angiographic catheter through which a hydrophilic guide wire was inserted and then manipulated into the renal collecting system. These maneuvers were monitored with fluoroscopy. Methylene blue or indigo carmine was administered intravenously to help identify the ureteral orifice when it was obscured by surrounding edema. A 5F, 12-14 cm double J stent was passed over the wire using both endoscopic and fluoroscopic guidance. The wire was subsequently removed and coiling of both ends of the stent was confirmed with fluoroscopy. The initial stent change interval was every 3 months and this was increased if there was no evidence of encrustation up to a maximum period of 6 months.

\section{RESULTS}

An average of 8.8 stent changes were performed per patient and the mean duration of ureteral stenting was 34.5 months (range 9.7-75.5). Which were the catheter caliber? How much time it remained in the place? Glomerular filtration rates were calculated by the Cockcroft-Gault formula and measured at baseline and at the time of the most recent stent change (Table-1). There was no significant decline in renal function during the period of stenting. There were no procedure related complications. One patient had periodic, recurrent urinary tract infections, which were managed with antibiotic therapy. Which were the symptoms related to double $\mathrm{J}$ ? It could be interesting to know about the costs per procedure and per patient/year. I think that it is elevated.

\section{COMMENTS}

Ureteral complications can develop after both cadaveric and living donor renal transplantation. They have been reported to occur in up to $10 \%$ of patients undergoing such procedures. Which was the incidence in your casuistic (1-3)? These complications include urine leak, ureteral necrosis, ureteral stricture and extrinsic obstruction from lymphocele. The underlying etiology may be due to surgical technique or compromised vascular integrity of the ureter. Some of these problems are transient and the patients can be successfully managed with antegrade or retrograde stent placement, or nephrostomy tube insertion $(4,5)$. Our cohort included patients who developed ureteral obstruction who had failed open surgical reconstruction or had long strictures and declined an open surgical repair. Why not? While other endourological approaches such as balloon dilation, endoureterotomy and cutting balloon incision have been used to manage patients developing ureteral obstruction after renal transplantation, our patient cohort were not thought to be good candidates for such treatments

Table 1 - Demographics and maintenance of glomerular filtration rates (GFR) in patients with renal allograft ureteral obstruction managed with chronic ureteral stent.

\begin{tabular}{lcccc}
\hline Patient & Age in Years & Number of Stent Changes & Baseline GFR & Most Recent GFR \\
\hline 1 & 61 & 19 & 55.9 & 57.9 \\
2 & 44 & 8 & 31.7 & 39.0 \\
3 & 53 & 8 & 58.6 & 60.4 \\
4 & 27 & 2 & 51.0 & 53.0 \\
5 & 67 & & 37.3 & 35.9 \\
\hline
\end{tabular}


based on the aforementioned factors. $(6,7)$. Why? Probably the costs of changing the double J frequently is more expensive than try a definitive endoscope procedure.

Our results indicate that repetitive stenting can be a successful form of management for this highly select group; those who fail open surgical reconstruction, those who are not thought to good candidates for definitive endourological therapy, and those who decline open surgical reconstruction. Which kind of patients? In the material and methods, the patients were not well characterized. Graft function was maintained at a mean follow-up of almost three years. There were no complications associated with the stent change procedures. Which were the criteria for choose the double J caliber? Which was the best caliber? Which caused fewer symptoms? It was used catheter shorter than the normal one?

There are great difference between 3 and 6 months. Which the used criterion to make the exchange in 3 or 6 months? The duration of stenting is a unique feature of our study. Patients were stented for a mean duration of almost 3 years and a range extending to 75 months. This is the longest reported duration of ureteral stenting in this population. The only comparable series was reported by Pappas and associates and the mean duration of stenting was 15 months with a range of 12 to 21 months (8).

We found that certain technical maneuvers facilitated stent placement when the ureteral orifice is located in the dome area of the bladder. Identification of the orifice when bladder inflammation or bullous edema is present may be aided by the intravenous administration of indigo carmine or methylene blue. This promotes the excretion of blue colored urine from the targeted orifice thus facilitating its identification. Furosemide may also be administered to promote a diuresis if ureteral efflux is not promptly identified with the latter measures. Alignment of the scope with the allograft ureteral orifice can be achieved with any of several maneuvers. The utilization of a $5 \mathrm{~F}$ angiographic catheter with a curved tip facilitates guide wire cannulation of the orifice. Manual suprapubic compression of the bladder may improve the alignment of an anteriorly located orifice with the angiographic catheter. Finally, flexible cystoscopy may also allow improved access to the orifice if these prior maneuvers are unsuccessful. The cannulation itself is aided by the use of a hydrophilic guidewire. Finally, we strongly recommend that fluoroscopy be used to monitor these procedures, as the course of the transplanted ureter may be variable.

\section{CONCLUSIONS}

Ideally, definitive correction of transplant graft ureteral obstruction with an open surgical or endourological approach should be considered for most patients with ureteral obstruction after renal transplantation. There is, however, a group of patients who either have failed these approaches or who are not candidates for them. This group was not studied and you cannot conclude on it. Repetitive ureteral stenting is a viable treatment option in this setting and may allow preservation of renal function.

\section{CONFLICT OF INTEREST}

None declared.

\section{REFERENCES}

1. Faenza A, Nardo B, Catena F, Scolari MP, d'Arcangelo GL, Buscaroli A, et al.: Ureteral stenosis after kidney transplantation. A study on 869 consecutive transplants. Transpl Int. 1999; 12: 334-40.

2. Shoskes DA, Hanbury D, Cranston D, Morris PJ: Urological complications in 1,000 consecutive renal transplant recipients. J Urol. 1995; 153: 18-21.

3. Kinnaert P, Hall M, Janssen F, Vereerstraeten P, Toussaint C, Van Geertruyden J: Ureteral stenosis after kidney transplantation: true incidence and long-term followup after surgical correction. J Urol. 1985; 133: 17-20.

4. Sigman DB, Del Pizzo JJ, Sklar GN: Endoscopic retrograde stenting for allograft hydronephrosis. J Endourol. 1999; 13: 21-5.

5. Bosma RJ, van Driel MF, van Son WJ, de Ruiter AJ, Mensink HJ: Endourological management of ureteral obstruction after renal transplantation. J Urol. 1996; 156: 1099-100. 
6. Kristo B, Phelan MW, Gritsch HA, Schulam PG: Treatment of renal transplant ureterovesical anastomotic strictures using antegrade balloon dilation with or without holmium:YAG laser endoureterotomy. Urology. 2003; 62: 831-4.

7. Schwartz BF, Chatham JR, Bretan P, Goharderakhshan R, Stoller ML: Treatment of refractory kidney trans- plant ureteral strictures using balloon cautery endoureterotomy. Urology. 2001; 58: 536-9.

8. Pappas P, Stravodimos KG, Adamakis I, Leonardou P, Zavos G, Constantinides C, et al.: Prolonged ureteral stenting in obstruction after renal transplantation: long-term results. Transplant Proc. 2004; 36: 1398-401.
Accepted after revision: January 25, 2006

\section{Correspondence address:}

Dr. Dean G. Assimos

Department of Urology

Wake Forest Univ. Sch. Med.

Medical Center Boulevard

Winston-Salem, NC 27157, USA

Fax: + 1336 716-5711

E-mail: dassimos@wfubmc.edu

\section{EDITORIAL COMMENT}

The authors describe their interesting experience with patients with ureteral stenosis after renal transplantation and treated by repetitive ureteral stenting. Among 460 grafts, 5 cases were included in the program $(1.08 \%)$. Three had failed to a previous reimplant surgery and 2 had refused a new surgical approach.

Ureteral stenosis occurs in 2 to $10 \%$ of renal grafts, being $80 \%$ in the uretero-vesical junction and $71 \%$ occurring in the first 3 months after transplantation (1). Lesions less than $2 \mathrm{~cm}$ of extension have better prognosis as well as those treated during the first 3 months post transplantation (2).

There are some proposals for the management of ureteral stenosis in graft patients, but the gold stan- dard approach is the re-anastomosis with the native ureter (3). However, the minimally invasive or endourological techniques have been considered to reduce the impact of this complication. Among these techniques, the balloon dilation, the balloon cutting endoureterotoy (Acucise ${ }^{\circledR}$ ), the Holmium laser endoureterotomy and the prolonged use of double- $\mathrm{J}$ stent are emphasized.

It is worthy to point out that the retrograde placement of ureteral stent can be challenging in kidney transplant patients. Nahas et al. described their experience with retrograde catheterization in patients with renal graft and ureteral dilation. In 9 of 12 cases (75\%), it was possible to perform successfully retrograde catheterization. The remaining cases were treated by percutaneous approach (4). 
Ureteral re-implant in kidney transplant patients is object of discussion in medical literature. Gauleria et al. compared the Leadbetter-Politano ureteral implant technique with the anterior ureteroneocystostomy and demonstrated with the second procedure a reduction in the stenosis index (7.7\% to $3.8 \%)$ (5).

Kristo and cols. described the technique of dilation of ureteral stenosis with a balloon, by anterior approach, in 9 kidney transplant patients. Six cases were treated exclusively with the balloon and in 3 cases the author included the Holmium laser for incision. He was successful in all cases, but all patients presented a stenosis of less than $0.5 \mathrm{~cm}$ extension (6).

Balloon cautery was used by Erturk et al. $\mathrm{He}$ reported an $86 \%$ resolution index, but severe bleeding was observed as a complication in one case (7).

The long-term use of ureteral catheter, described by Pappas et al., is promising. The stents were replaced each 3 months and remained for a median time of 15 months. The authors reported $90 \%$ of cure of ureteral stenosis in kidney transplant patients (8).

In this issue of the International Braz J Urol the authors contribute to the experience with periodical replacement of ureteral catheter for the treatment of ureteral stenosis in transplanted patients. In this study, the use of double-J stent allowed the preservation of renal function in all cases.

\section{REFERENCES}

1. Shoskes DA, Hanbury D, Cranston D, Morris PJ: Urological complications in 1,000 consecutive renal transplant recipients. J Urol. 1995; 153: 18-21.

2. Goldfischer ER, Gerber GS: Endoscopic management of ureteral strictures. J Urol. 1997; 157: 770-5.

3. Schwartz BF, Chatham JR, Bretan P, Goharderakhshan R, Stoller ML: Treatment of refractory kidney transplant ureteral strictures using balloon cautery endoureterotomy. Urology. 2001; 58: 536-9.

4. Nahas WC, Gil AO, Mazzucchi E, et al. Retrograde pyelography in transplant kidney: a reliable method. Urol Panamericana. 1995; 7: 39-40.

5. Guleria S, Chahal R, Madaan S, Irving HC, Newstead CG, Pollard SG, et al.: Ureteric complications of renal transplantation: the impact of the double $\mathrm{J}$ stent and the anterior extravesical ureteroneocystostomy. Transplant Proc. 2005; 37: 1054-6.

6. Kristo B, Phelan MW, Gritsch HA, Schulam PG: Treatment of renal transplant ureterovesical anastomotic strictures using antegrade balloon dilation with or without holmium:YAG laser endoureterotomy. Urology. 2003; 62: 831-4.

7. Erturk E, Burzon DT, Waldman D: Treatment of transplant ureteral stenosis with endoureterotomy. J Urol. 1999; 161: 412-4.

8. Pappas P, Stravodimos KG, Adamakis I, Leonardou P, Zavos G, Constantinides C, et al.: Prolonged ureteral stenting in obstruction after renal transplantation: longterm results. Transplant Proc. 2004; 36: 1398-401.

Dr. Ricardo Jordão Duarte Assistant Professor of Urology University of Sao Paulo, SP, Brazil E-mail: ricjordao@uol.com.br 